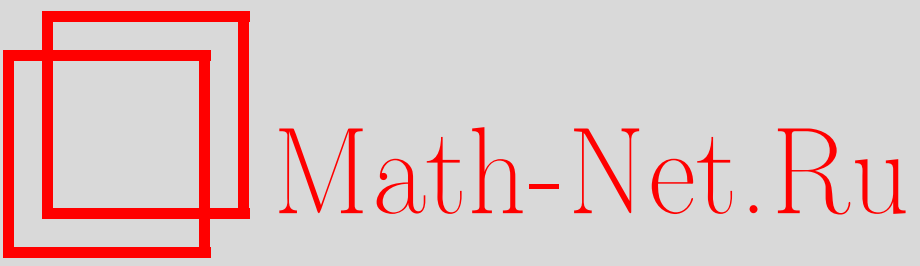

И. В. Широков, Координаты Дарбу на $K$-орбитах и спектры операторов Казимира на группах Ли, ТМФ, 2000, том 123, номер 3, 407-423

DOI: https://doi.org/10.4213/tmf612

Использование Общероссийского математического портала Math-Net.Ru подразумевает, что вы прочитали и согласны с пользовательским соглашением

http://www.mathnet.ru/rus/agreement

Параметры загрузки:

IP : 54.237 .59 .107

26 апреля 2023 г., 13:43:54 
ТЕОРЕТИЧЕСКАЯ

И МАТЕМАТИЧЕСКАЯ

ФИЗИКА

Том 123, № 3

июнь, 2000

(C) 2000 r.

И.В. Широков*

\title{
КООР ДИНАТЫ ДАРБУ НА $K$-ОРБИТАХ И СПЕКТРЫ ОПЕРАТОРОВ КАЗИМИРА НА ГРУППАХ ЛИ
}

\begin{abstract}
Предложен алгоритм получения спектров операторов Казимира (Лапласа) на группе Ли. Доказано, что существование нормальной поляризации для линейного функционала на алгебре Ли является необходимым и достаточным условием для линейного по "импульсам" перехода к локальным каноническим координатам Дарбу $(p, q)$ на орбите коприсоединенного представления. Показано, что $\lambda$-представление алгебры Ли, которое используется, в частности, в методах интегрирования дифференциальных уравнений, есть результат квантования скобки Пуассона на коалгебре Ли в канонических координатах.
\end{abstract}

\section{ВВЕДЕНИЕ}

Метод орбит, открытьй в пионерских работах Кириллова $[1,2]$ (см. также $[3,4])$, является универсальной базой для проведения гармонического анализа на однородных пространствах и построения на этой основе новых методов интегрирования линейных дифференциальных уравнений. Предварительный вариант этой программы приведен в работе [5].

Основным результатом настояшей статьи является алгоритм получения методами линейной алгебры спектров операторов Казимира (Лапласа) на группе Ли по известным структурным константам и информации о компактности определенных подгрупп. Показано, что сушествование нормальной поляризации для линейного функционала $\lambda$ необходимое и достаточное условие для линейного по "импульсам" перехода к локальным каноническим координатам Дарбу $(p, q)$ на $K$-орбите $O_{\lambda}$. (Автором разработана компьютерная программа в системе аналитических вычислений "Maple-V", строящая по заданному функционалу и его нормальной поляризации канонические координаты Дарбу.) Последуюшее "квантование" приводит к понятию $\lambda$-представления алгебр Ли - сопоставление $K$-орбите специального представления алгебры Ли дифференшиальными операторами. Впервые $\lambda$-представление появилось в методе некоммутативного интегрирования линейных дифференциальных уравнений [6] как "квантовый” аналог метода некоммутативного интегрирования конечномерных гамильтоновых систем Ми-

\footnotetext{
* Омский государственный университет, Омск, Россия. E-mail: shirokov@univer.omsk.su
} 
щенко и Фоменко [7]. Операторы $\lambda$-представления присутствуют также неявным образом как генераторы неприводимых представлений групп Ли.

\section{1. ОПИСАНИЕ $K$-ОРБИТ}

Пусть $G$ - вешественная связная $n$-мерная группа Ли, $\mathcal{G}$ - ее алгебра Ли. Действие присоединенного представления $A d^{*}$ группы Ли на дуальном пространстве $\mathcal{G}^{*}$ расслаивает последнее на четномерные орбиты ( $K$-орбиты). Максимальная размерность $K$-орбит равна $n-r$, где число $r$ назьвается индексом (ind $\mathcal{G}$ ) алгебры Ли и определяется как размерность аннулятора ковектора обшего положения. Будем говорить, что линейньй функционал (ковектор) $\lambda$ имеет степень вырождения $s$, если он принадлежит $K$-орбите $O_{\lambda}$ размерности $\operatorname{dim} O_{\lambda}=n-r-2 s(s=0, \ldots,(n-r) / 2)$.

Разложим пространство $\mathcal{G}^{*}$ в сумму непересекаюшихся инвариантных алгебраических поверхностей $M_{s}$, состояших из $K$-орбит одинаковой размерности. Это можно сделать следуюшим образом. Обозначим через $f_{i}$ координаты ковектора $f$ в дуальном базисе: $f=f_{i} e^{i},\left\langle e^{i}, e_{j}\right\rangle=\delta_{j}^{i}$ (здесь $\left\{e_{j}\right\}$ - базис алгебры $\mathcal{G}$ ). Векторные поля на $\mathcal{G}^{*}$

$$
Y_{i}(f) \equiv C_{i j}(f) \frac{\partial}{\partial f_{j}}, \quad C_{i j}(f) \equiv C_{i j}^{k} f_{k},
$$

являются генераторами группы преобразований $G$, действуюшей на пространстве $\mathcal{G}^{*}$, и поэтому их линейная оболочка образует касательное пространство $T_{f} O_{\lambda}$ к орбите $O_{\lambda}$, содержашей точку $f$. Таким образом, размерность орбиты $O_{\lambda}$ определяется рангом матрицы $C_{i j}$ :

$$
\operatorname{dim} O_{\lambda}=\operatorname{rank} C_{i j}(\lambda)
$$

Нетрудно проверить, что ранг матришы $C_{i j}$ постоянен на орбите. Таким образом, приравнивая к нулю соответствуюшие миноры матрицы $C_{i j}(f)$ и "не разрешая" обращаться в нуль минорам меньшего порядка, мы получим полиномиальные уравнения, определяющие поверхности $M_{s}$ :

$$
\begin{aligned}
M_{0} & =\left\{f \in \mathcal{G}^{*} \mid \neg\left(F^{1}(f)=0\right)\right\}, \\
M_{s} & =\left\{f \in \mathcal{G}^{*} \mid F^{s}(f)=0, \neg\left(F^{s+1}(f)=0\right)\right\}, \quad s=1, \ldots, \frac{n-r}{2}-1, \\
M_{\frac{n-r}{2}} & =\left\{f \in \mathcal{G}^{*} \mid F^{\frac{n-r}{2}}(f)=0\right\} .
\end{aligned}
$$

Здесь через $F^{s}(f)$ обозначена совокупность всех миноров матрицы $C_{i j}(f)$ размера $n-$ $r-2 s+2$, условие $F^{s}(f)=0$ означает, что все миноры матрицы $C_{i j}(f)$ размера $n-r-$ $2 s+2$ в точке $f$ обрашаются в нуль; $\neg\left(F^{s}(f)=0\right)$ означает, что в точке $f$ соответствующие миноры не обрашаются в нуль одновременно.

Пространства $M_{s}$ можно также определить как множество точек $f$, в которых все поливекторы степени $n-r-2 s+1$ вида $Y_{i_{1}}(f) \wedge \cdots \wedge Y_{i_{n-r-2 s+1}}(f)$ обращаются в нуль, но не все поливекторы степени $n-r-2 s-1$ равны нулю.

Отметим, что в общем случае поверхность $M_{s}$ состоит из нескольких непересекаюшихся инвариантных компонент, которые мы будем различать буквенными индексами 
$M_{s}=M_{s a} \cup M_{s b} \ldots$. (Чтобы каждый раз отдельно не оговаривать случай, когда пространство $M_{s}$ несвязно, примем следуюшее соглашение: число $s$ в круглых скобках $(s)$ будет обозначать конкретный тип для орбиты со степенью вырождения $s$.) Каждая компонента $M_{(s)}$ определяется своим набором однородных полиномов $F_{\alpha}^{(s)}(f)$, удовлетворяющих условиям

$$
\left.Y_{i} F_{\alpha}^{(s)}(f)\right|_{F^{(s)}(f)=0}=0 .
$$

Инвариантные алгебраические поверхности $M_{(s)}$ не являются линейными пространствами, но тем не менее являются звездными множествами, т.е. если $f \in M_{(s)}$, то $t f \in$ $M_{(s)}, t \in R^{1}$.

ПримеР 1 (Группа Пуанкаре $\left.P^{1,3}\right)$. Группа Пуанкаре $P^{1,3}=T^{4} \triangleright S O(1,3)$ является группой движений пространства Минковского и представляет собой полупрямое произведение полупростой группы вращений Лоренца $S O(1,3)$ и четырехмерной коммутативной группы сдвигов $T^{4}$. Коммутационные соотношения алгебры Пуанкаре $\mathcal{P}^{1,3}=$ $\left\{e_{a b}, e_{a}\right\}$ можно представить следуюшим образом:

$$
\begin{aligned}
{\left[e_{a b}, e_{c d}\right] } & =g_{a d} e_{b c}-g_{a c} e_{b d}+g_{b c} e_{a d}-g_{b d} e_{a c}, \quad\left[e_{a}, e_{b c}\right]=g_{a b} e_{c}-g_{a c} e_{b} \\
{\left[e_{a}, e_{b}\right] } & =0, \quad a, b, c, d=0,1,2,3, \quad g_{a b}=\operatorname{diag}(1,-1,-1,-1) .
\end{aligned}
$$

В этом примере $n=10, r=2$. Обозначая через $\left\{l_{a b}, p_{a}\right\}$ координаты ковектора $f$ в дуальном базисе, приведем явный вид поверхностей $M_{(s)}$ :

$$
\begin{aligned}
\left(\mathcal{P}^{1,3}\right)^{*} & =M_{0} \cup M_{1 a} \cup M_{1 b} \cup M_{2} \cup M_{4}, \\
M_{0} & =\left\{f \in R^{10} \mid \neg\left(W_{a} p_{b}-W_{b} p_{a}=0\right)\right\}, \quad \operatorname{dim} M_{0}=10, \\
M_{1 a} & =\left\{f \in R^{10} \mid W_{a}=0, \neg\left(p_{a}=0\right)\right\}, \quad \operatorname{dim} M_{1 a}=7, \\
M_{1 b} & =\left\{f \in R^{10} \mid W_{a} p_{0}=W_{0} p_{a}, \neg\left(W_{a}=0\right)\right\}, \quad \operatorname{dim} M_{1 b}=7, \\
M_{2} & =\left\{f \in R^{10} \mid p_{a}=0, \neg(f=0)\right\}, \quad \operatorname{dim} M_{2}=6, \\
M_{3} & =\varnothing, \quad M_{4}=\{f=0\}, \quad \operatorname{dim} M_{4}=0 .
\end{aligned}
$$

(Здесь введено обозначение $W^{a} \equiv(1 / 2) \varepsilon^{a b c d} l_{b c} p_{d}$, а поднятие и опускание индекса осуществляется с помощью диагональной матрицы $g_{a b}$.) Отметим, что в силу тождества $W^{a} p_{a}=0$ из четырех функций $W^{a}$ лишь три являются независимыми.

На дуальном пространстве $\mathcal{G}^{*}$ определена вырожденная линейная скобка Пуассона

$$
\{\varphi, \psi\}(f) \equiv\langle f,[\nabla \varphi(f), \nabla \psi(f)]\rangle, \quad \varphi, \psi \in C^{\infty}\left(\mathcal{G}^{*}\right) .
$$

Непостоянные на $M_{(s)}$ функции $K_{\mu}^{(s)}(f)$ называются функииями Казимира $(s)$-типа, если они коммутируют с любой функцией на $M_{(s)}$.

Функции Казимира $(s)$-типа находятся из уравнений

$$
\left.C_{i j}(f) \frac{\partial K_{\mu}^{(s)}(f)}{\partial f_{j}}\right|_{f \in M_{(s)}}=0, \quad i=1, \ldots, n .
$$


Очевидно, что число $r_{(s)}$ независимых функций Казимира $(s)$-типа связано с размерностью пространства $M_{(s)}: r_{(s)}=\operatorname{dim} M_{(s)}+2 s+r-n$. Так как пространства $M_{(s)}$ звездные, то без потери обшности можно считать, что функции Казимира $K_{\mu}^{(s)}(f)$ однородны:

$$
\frac{\partial K_{\mu}^{(s)}(f)}{\partial f_{i}} f_{i}=m_{\mu}^{(s)} K_{\mu}^{(s)}(f) \Longleftrightarrow K_{\mu}^{(s)}(t f)=t^{m_{\mu}^{(s)}} K_{\mu}^{(s)}(f), \quad \mu=1, \ldots, r_{(s)} .
$$

В общем случае функции Казимира являются многозначными (например, если пространство орбит $\mathcal{G}^{*} / G$ не полуотделимо, то функции Казимира бесконечнозначные). В дальнейшем под функцией Казимира мы будем понимать некоторую фиксированную ветвь многозначной функции $K_{\mu}^{(s)}$. При этом функции Казимира $K_{\mu}^{(s)}$ в общем случае будут лишь локальными инвариантами коприсоединенного представления, т.е. равенство $K_{\mu}^{(s)}\left(A d_{g}^{*} f\right)=K_{\mu}^{(s)}(f)$ имеет место для элементов $g$, принадлежаших некоторой окрестности единицы группы $G$.

ЗАмечАниЕ 1 . Не вдаваясь в детали, отметим, что пространства $M_{(s)}$ являются критическими поверхностями для некоторых полиномиальных функций Казимира типа $(s-1)$. Это замечание дает простой и конструктивный способ построения функций $F^{(s)}$

Обозначим через $\Omega^{(s)} \subset R^{r(s)}$ множество значений отображения $K^{(s)}: M_{(s)} \rightarrow R^{r}(s)$ и введем локально-инвариантное подмножество $O_{\omega}^{(s)}$ - поверхность уровня

$$
O_{\omega}^{(s)}=\left\{f \in M_{(s)} \mid K_{\mu}^{(s)}(f)=\omega_{\mu}^{(s)}, \quad \mu=1, \ldots, r_{(s)} ; \omega^{(s)} \in \Omega^{(s)}\right\} .
$$

Размерность множества $O_{\omega}^{(s)}$ совпадает с размерностью орбиты $O_{\lambda} \in M_{(s)}$, где $\omega^{(s)}=$ $K^{(s)}(\lambda)$. Если функции Казимира однозначны, то пространство орбит отделимо и в этом случае множество $O_{\omega}^{(s)}$ состоит из счетного (как правило, конечного) числа орбит, вследствие этого поверхность уровня мы будем называть классом орбит.

Таким образом, пространство $\mathcal{G}^{*}$ состоит из объединения связных инвариантных непересекающихся алгебраических поверхностей $M_{(s)}$, которые, в свою очередь, представляют собой объединение классов орбит $O_{\omega}^{(s)}$ :

$$
\mathcal{G}^{*}=\bigcup_{(s)} M_{(s)}=\bigcup_{(s)} \bigcup_{\omega(s) \in \Omega^{(s)}} O_{\omega}^{(s)} .
$$

ПримеР 2 (Группа Пуанкаре $P^{1,3}$, продолжение примера 1). Приведем для этого случая разложение $(1.4)\left(\Delta_{1,3} \equiv p_{a} p^{a}\right)$ :

$$
\begin{aligned}
\left(\mathcal{P}^{1,3}\right)^{*} & =\left(\bigcup_{\omega \in R^{2}} O_{\omega}^{0}\right) \cup\left(\bigcup_{\omega \in R^{1}} O_{\omega}^{1 a}\right) \cup\left(\bigcup_{\omega \in R^{1} \backslash\{0\}} O_{\omega}^{1 b}\right) \cup\left(\bigcup_{\omega \in R^{2}} O_{\omega}^{2}\right) \cup\{0\}, \\
O_{\omega}^{0} & =\left\{f \in M_{0} \mid W^{a} W_{a}=\omega_{1}^{0}, \quad \Delta_{1,3}=\omega_{2}^{0}\right\}, \quad \Omega^{0}=R^{2}, \\
O_{\omega}^{1 a} & =\left\{f \in M_{1 a} \mid \Delta_{1,3}=\omega_{1}^{1 a}\right\}, \quad \Omega^{1 a}=R^{1}, \\
O_{\omega}^{1 b} & =\left\{f \in M_{1 b} \mid \frac{W_{0}}{p_{0}}=\omega_{1}^{1 b}\right\}, \quad \Omega^{1 b}=R^{1} \backslash\{0\}, \\
O_{\omega}^{2} & =\left\{f \in M_{2} \mid l_{a b} l^{a b}=\omega_{1}^{2}, \quad \varepsilon^{a b c d} l_{a b} l_{c d}=\omega_{2}^{2}\right\}, \quad \Omega^{2}=R^{2} .
\end{aligned}
$$


В этом примере каждый класс состоит из нескольких $K$-орбит (от одной до четырех в зависимости от знаков параметров $\left.\omega^{(s)}\right)$.

Рассмотрим фактор-пространство $B_{(s)}=M_{(s)} / G$, точками которого являются орбиты $O_{\lambda} \in M_{(s)}$. Очевидно, что $\operatorname{dim} B_{(s)}=r_{(s)}$. Введем локальные координаты $j$ на $B_{(s)}$. Для этого параметризуем $(s)$-ковектор $\lambda \in M_{(s)}$ вешественными параметрами $j=\left(j_{1}, \ldots, j_{r_{(s)}}\right)$, полагая, что $\lambda$ зависит от $j$ линейным образом (это можно сделать в силу звездности поверхности $\left.M_{(s)}\right)$ :

$$
\lambda=\lambda(j)
$$

причем

$$
F_{\alpha}^{(s)}(\lambda(j)) \equiv 0, \quad K_{\mu}^{(s)}(\lambda(j))=\omega_{\mu}^{(s)}(j), \quad \operatorname{det} \frac{\partial \omega_{\mu}^{(s)}(j)}{\partial j_{\nu}} \neq 0 .
$$

Иначе говоря, $\lambda(j)$ - локальное сечение расслоения $M_{(s)} \rightarrow B_{(s)}$. Определим прообраз $\Theta_{(s)} \equiv\left(\omega^{(s)}\right)^{-1}\left(\Omega^{(s)}\right) \subset R^{r(s)}$ и дискретную группу $\Gamma^{(s)}$ преобразований множества $\Theta_{(s)}: j \rightarrow \hat{j}$ так, что $\omega_{\mu}^{(s)}(\hat{j})=\omega_{\mu}^{(s)}(j)$. Тогда каждой точке $j$ из области $J^{(s)} \equiv$ $\Theta_{(s)} / \Gamma^{(s)} \subset R^{r(s)}$ соответствует один класс $O_{\omega}^{(s)}$.

Простейшие примеры показывают, что глобальной параметризации на всем пространстве $M_{(s)}$ в общем случае не существует, т.е. многообразие $B_{(s)}$ не покрывается одной картой. В этом случае определим атлас карт на $B_{(s)}$ и введем параметризацию на соответствуюших связных инвариантных непересекаюшихся подмножествах $M_{(s)}^{A}, M_{(s)}^{B}, \ldots$, имеюших в $M_{(s)}$ ненулевую меру, следуюшим образом:

$$
M_{(s)}=M_{(s)}^{A} \cup M_{(s)}^{B} \cup \cdots .
$$

При этом соответствуюшие области значений $J^{A}, J^{B}, \ldots$ параметров $j$ удовлетворяют соотношению $\Omega^{(s)}=\omega^{(s)}\left(J^{A}\right) \cup \omega^{(s)}\left(J^{B}\right) \cup \cdots$.

Ниже будет показано, что каждому пространству $M_{(s)}^{A}$ соответствует свой тип спектра операторов Казимира на группе Ли. Поэтому мы можем говорить, что разложение (1.5) - это разложение по спектральному типу и $(s)$-орбита $O_{\lambda}$ принадлежит спектральному типу $A$, если $O_{\lambda} \in M_{(s)}^{A}$. Проиллюстрируем разложение (1.5) на простом примере.

ПримеР 3 (Группа $S O(2,1))$. В этом случае $\left[e_{1}, e_{2}\right]=e_{2},\left[e_{2}, e_{3}\right]=2 e_{1},\left[e_{3}, e_{1}\right]=e_{3}$. Разложение (1.4) имеет вид

$$
O_{\omega}^{0}=\left\{f_{1}^{2}+f_{2} f_{3}=\omega, \neg(f=0)\right\}, \quad O^{1}=\{f=0\} .
$$

При $\omega>0$ класс $O_{\omega}^{0}$ состоит из двух орбит. Для невырожденных орбит $\Omega=R^{1}$. Единой параметризации в этом случае не сушествует. Действительно, самый общий вид параметризации $\lambda(j)=\left(a_{1} j, a_{2} j, a_{3} j\right)$ (здесь $a_{i}$ - некоторые числа) приводит к равенству $\omega(j)=a j^{2}$, где $a=a_{1}^{2}+a_{2} a_{3}$, и поэтому (в зависимости от знака числа $\left.a\right) \omega(j)$ 
всегда либо больше нуля, либо меньше, либо равно нулю, т.е. $\omega\left(R^{1}\right) \neq \Omega$. Ведем два спектральных типа:

$$
\begin{array}{lll}
\text { тип А : } \lambda(j)=(0, j, j), & J^{\mathrm{A}}=[0, \infty), & O_{\omega(j)}^{0 \mathrm{~A}}=\left\{f_{1}^{2}+f_{2} f_{3}=j^{2}, f \neq 0\right\}, \\
\text { тип В : } \lambda(j)=(0, j,-j), & J^{\mathrm{B}}=(0, \infty), & O_{\omega(j)}^{0 \mathrm{~B}}=\left\{f_{1}^{2}+f_{2} f_{3}=-j^{2}, f \neq 0\right\} .
\end{array}
$$

Позже мы вернемся к этому примеру и покажем, что тип А соответствует непрерывному, а тип В - дискретному спектрам оператора Лапласа (Казимира) на группе $S O(2,1)$.

Полученная выше классификация $K$-орбит позволяет более детально описать структуру аннулятора $\mathcal{G}_{\lambda}$ произвольного, в обшем случае вырожденного, ковектора $\lambda$.

Пусть $\lambda$-некоторый ковектор $(s)$-типа. Из определения аннулятора следует $\operatorname{dim} \mathcal{G}_{\lambda}=$ $\operatorname{corank} C_{i j}(\lambda)=\operatorname{codim} O_{\lambda}=2 s+r$.

Можно проверить, что функции $\Phi_{a}^{(s)}(f)=\left(F_{\alpha}^{(s)}(f), K_{\mu}^{(s)}(f)\right)(a=1, \ldots, 2 s+r)$ коммутируют между собой относительно скобки Пуассона следующим образом:

$$
\begin{aligned}
\left\{F_{\alpha}^{(s)}, F_{\beta}^{(s)}\right\}(f) & =C_{\alpha \beta}^{\gamma}(f) F_{\gamma}^{(s)}(f), \\
\left\{F_{\alpha}^{(s)}, K_{\mu}^{(s)}\right\}(f) & =C_{\alpha \mu}^{\beta}(f) F_{\beta}^{(s)}(f), \\
\left\{K_{\mu}^{(s)}, K_{\nu}^{(s)}\right\}(f) & =C_{\mu \nu}^{\alpha}(f) F_{\alpha}^{(s)}(f) .
\end{aligned}
$$

Из независимости функций $\Phi_{a}^{(s)}(f)$, а также определения пространства $M_{(s)}$ следует, что градиенты $\nabla \Phi^{(s)}(\lambda)$ линейно независимы и составляют базис $(2 s+r)$-мерной алгебры Ли $\mathcal{G}_{\lambda}$ с коммутационными соотношениями (для краткости записи мы индекс $(s)$ опускаем):

$$
\begin{aligned}
{\left[\nabla F_{\alpha}(\lambda), \nabla F_{\beta}(\lambda)\right] } & =C_{\alpha \beta}^{\gamma}(\lambda) \nabla F_{\gamma}(\lambda), \\
{\left[\nabla F_{\alpha}(\lambda), \nabla K_{\mu}(\lambda)\right] } & =C_{\alpha \mu}^{\beta}(\lambda) \nabla F_{\beta}(\lambda), \\
{\left[\nabla K_{\mu}(\lambda), \nabla K_{\nu}(\lambda)\right] } & =C_{\mu \nu}^{\alpha}(\lambda) \nabla F_{\alpha}(\lambda) .
\end{aligned}
$$

Из коммутационных соотношений (1.6) следует

УТВЕРЖДЕНИЕ 1. Аннулятор $\mathcal{G}_{\lambda}$ произвольного $(s)$-ковектора $\lambda$ содержит идеaл $\mathcal{N}_{\lambda}=\left\{\nabla F_{\alpha}^{(s)}(\lambda)\right\}$, а фактор-алгебра $\mathcal{K}_{\lambda}=\mathcal{G}_{\lambda} / \mathcal{N}_{\lambda}=\left\{\nabla K_{\mu}^{(s)}(\lambda)+\mathcal{N}_{\lambda}\right\}$ коммутативна и $r_{(s)}$-мерна.

Замкнутые подгруппы группы $G$ с алгебрами Ли $\mathcal{G}_{\lambda}, \mathcal{N}_{\lambda}, \mathcal{K}_{\lambda}$ мы будем обозначать соответственно через $G_{\lambda}, N_{\lambda}$ и $K_{\lambda}=G_{\lambda} / N_{\lambda}$.

\section{2. КООР ДИНАТЫ ДАРБУ, $\lambda$-ПРЕДСТАВЛЕНИЯ АЛГЕБР ЛИ}

Обозначим через $\omega_{\lambda}$ форму Кириллова, которая определяет на орбите $O_{\lambda}$ симплектическую структуру и на касательных векторах $a, b$ к орбите действует следующим образом:

$$
\omega_{\lambda}(a, b)=\langle\lambda,[\alpha, \beta]\rangle, \quad \text { где } a=a d_{\alpha}^{*} \lambda, \quad b=a d_{\beta}^{*} \lambda
$$


Ограничение скобки Пуассона (1.2) на орбиту совпадает со скобкой Пуассона, порожденной симплектической формой $\omega_{\lambda}$. Согласно известной теореме Дарбу на орбите $O_{\lambda}$ сушествуют локальные канонические координаты (координаты Дарбу), в которых форма $\omega_{\lambda}$ имеет вид

$$
\omega_{\lambda}=d p_{a} \wedge d q^{a}, \quad a=1, \ldots, \frac{1}{2} \operatorname{dim} O_{\lambda}=\frac{n-r}{2}-s,
$$

где $s$ - степень вырождения орбиты.

Пусть $\lambda$ - некоторый ковектор $(s)$-типа и $f \in O_{\lambda}$. Нетрудно видеть, что переход к каноническим координатам Дарбу $\left(f_{i}\right) \rightarrow\left(p_{a}, q^{a}\right)$ состоит в построении аналитических функций $f_{i}=f_{i}(q, p, \lambda)$ от переменных $(p, q)$, удовлетворяющих следующим условиям:

$$
\begin{gathered}
f_{i}(0,0, \lambda)=\lambda_{i} \\
\frac{\partial f_{i}(q, p, \lambda)}{\partial p_{a}} \frac{\partial f_{j}(q, p, \lambda)}{\partial q^{a}}-\frac{\partial f_{j}(q, p, \lambda)}{\partial p_{a}} \frac{\partial f_{i}(q, p, \lambda)}{\partial q^{a}}=C_{i j}^{k} f_{k}(q, p, \lambda) \\
F_{\alpha}^{(s)}(f(q, p, \lambda))=0, \quad K_{\mu}^{(s)}(f(q, p, \lambda))=K_{\mu}^{(s)}(\lambda) .
\end{gathered}
$$

Потребуем, чтобы переход к каноническим координатам (или иначе $q p$-переход) был линейным по переменным $p_{a}$ :

$$
f_{i}(q, p, \lambda)=\alpha_{i}^{a}(q) p_{a}+\chi_{i}(q, \lambda), \quad \operatorname{rank} \alpha_{i}^{a}(q)=\frac{1}{2} \operatorname{dim} O_{\lambda}
$$

Конечно, в общем случае перехода (2.4) не существует, но если допустить, что $\alpha_{i}^{a}(q)$ и $\chi_{i}(q, \lambda)$ являются голоморфными функциями от комплексных переменных $q$, то тем самым мы значительно расширим класс алгебр Ли и $K$-орбит, для которых этот переход сушествует. (Считаем, что функционалы из $\mathcal{G}^{*}$ продолжаются на $\mathcal{G}^{c}$ по линейности.) По-видимому, для произвольной алгебры Ли и любой ее невырожденной орбиты переход (2.4) существует.

ТЕОРема 1. Линейный переход к каноническим координатам на орбите $O_{\lambda}$ существует тогда и только тогда, когда для линейного функчионала $\lambda$ существует нормальная, в общем случае комплексная, поляризация, т.е. подалгебра $\mathcal{H} \subset \mathcal{G}^{c}$ такая, что

$$
\operatorname{dim} \mathcal{H}=n-\frac{1}{2} \operatorname{dim} O_{\lambda}, \quad\langle\lambda,[\mathcal{H}, \mathcal{H}]\rangle=0, \quad \lambda+\mathcal{H}^{\perp} \subset O_{\lambda}
$$

Прежде чем переходить к обсуждению и доказательству этой теоремы, сделаем отступление, имеющее, на наш взгляд, и самостоятельную ценность.

Пусть $X_{i}(x)=X_{i}^{a}(x) \partial_{x^{a}}$ - генераторы группы преобразований, образующие $n$-мерную алгебру Ли $\mathcal{G}$ векторных полей на однородном пространстве $M=G / H:\left[X_{i}, X_{j}\right]=$ $C_{i j}^{k} X_{k}$ (здесь и далее $x^{a}(a=1, \ldots, m=\operatorname{dim} M)$ - локальные координаты точки $\left.x \in M\right)$; $H$ - группа изотропии отмеченной точки $x_{0}, \mathcal{H}$ - ее алгебра Ли. Неоднородные операторы первого порядка $\widetilde{X}_{i}=X_{i}+\chi_{i}(x)$ будем называть продолжением генераторов 
$X_{i}$, если они по-прежнему удовлетворяют коммутационным соотношениям алгебры $\mathcal{G}$ $\left(\chi_{i}(x)\right.$ - гладкие функции на $\left.M\right)$.

По определению $n$-компонентная функция $\chi(x)$ находится из системы уравнений

$$
X_{i}^{a}(x) \frac{\partial \chi_{j}(x)}{\partial x^{a}}-X_{j}^{a}(x) \frac{\partial \chi_{i}(x)}{\partial x^{a}}=C_{i j}^{k} \chi_{k}(x)
$$

Решения этой системы составляют линейное пространство, в котором можно выделить модуль тривиальных решений вида

$$
\chi^{0}=\left\{\chi_{i}^{0}(x)=X_{i}^{a}(x) \frac{\partial S(x)}{\partial x^{a}}\right\}
$$

где $S(x)$ - произвольная гладкая функция. Построение тривиальных продолжений $\widetilde{X}_{i}=$ $e^{-S} X_{i} e^{S}$ эквивалентно “калибровочному" преобразованию $\partial_{x^{a}} \rightarrow \partial_{x^{a}}+\partial_{x^{a}} S(x)$. В дальнейшем нас будут интересовать лишь нетривиальные продолжения, образующие фактор-пространство всех решений системы (2.5) по модулю тривиальных решений.

УТВЕРЖДЕНИЕ 2. Пространство нетривиальных продолжсений конечномерно и изоморфно фактор-пространству $\mathcal{H}^{*} /[\mathcal{H}, \mathcal{H}]^{*}$.

Здесь мы не будем приводить полного доказательства этого утверждения, однако приведем явный вид всех нетривиальных решений системы (2.5), откуда и будет видна справедливость утверждения 2.

Произведем перенумерацию и замену базиса в алгебре $\mathcal{G}$ :

$$
X_{a}\left(x_{0}\right)=\left.\frac{\partial}{\partial x^{a}}\right|_{x_{0}}, \quad a=1, \ldots, m, \quad X_{\alpha}\left(x_{0}\right)=0, \quad \alpha=m+1, \ldots, n
$$

Будем считать, что действие группы $G$ на $M$ правое. Произвольный элемент $g \in G$ представляется в виде $g=h s(x)$, где $h \in H, s(x)$ - гладкое борелевское отображение $M \rightarrow G$, сопоставляющее каждой точке $x$ пространства $M$ правый смежный класс $H s(x) \subset G$. В координатах $g=\left(h^{\alpha}, x^{a}\right)$ (считаем, что $e=\left(0, x_{0}\right)$ ) левоинвариантные векторные поля $\xi_{i}(g)$ имеют вид $\xi_{i}(g)=X_{i}^{a}(x) \partial_{x^{a}}+\xi_{i}^{\alpha}(h, x) \partial_{h^{\alpha}}$. Прямым выгислением можно проверить, что операторы

$$
\tilde{X}_{i}=X_{i}+\xi_{i}^{\alpha}(0, x) \lambda_{\alpha}, \quad \lambda \in \mathcal{H}^{*}, \quad\langle\lambda,[\mathcal{H}, \mathcal{H}]\rangle=0
$$

являются нетривиальными продолжениями, и можно показать, что других нетривиальных продолжений не сушествует.

Изложим теперь основные моменты доказательства теоремы 1. 
ДокаЗАТЕЛЬСтво теОРемы 1. Распишем подробнее с учетом (2.4) формулу (2.2):

$$
\begin{aligned}
\alpha_{i}^{a}(q) \partial_{q^{a}} \alpha_{j}^{b}(q)-\alpha_{j}^{a}(q) \partial_{q^{a}} \alpha_{i}^{b}(q) & =C_{i j}^{k} \alpha_{k}^{b}(q), \\
\alpha_{i}^{a}(q) \partial_{q^{a}} \chi_{j}(q, \lambda)-\alpha_{j}^{a}(q) \partial_{q^{a}} \chi_{i}(q, \lambda) & =C_{i j}^{k} \chi_{k}(q, \lambda) .
\end{aligned}
$$

Формула (2.6) эквивалентна выражению $\left[a_{i}, a_{j}\right]=C_{i j}^{k} a_{k}$, где $a_{i}=\alpha_{i}^{a}(q) \partial_{q^{a}}$, и поэтому операторы $a_{i}$ являются генераторами группы преобразований, действуюшей в области $Q\left(Q=G / H\right.$ для вешественной поляризации и $Q=G^{c} / H$ для комплексной поляризации, здесь $H-$ группа Ли алгебры $\mathcal{H})$. Так как $\operatorname{rank} \alpha_{i}^{a}(q)=(1 / 2) \operatorname{dim} O_{\lambda}=\operatorname{dim} Q$, справедливо утверждение: если существуют решения системы (2.6), то существует алгебра изотропии $\mathcal{H}$ точки $q=0$ размерности $n-(1 / 2) \operatorname{dim} O_{\lambda}$. Очевидно, что верно и обратное: наличие $n-\operatorname{dim} Q$-мерной подалгебры $\mathcal{H}$ достаточно для сушествования решений системы (2.6).

Как следует из утверждения 2 , сушествование решений системы $(2.7)$ с начальными условиями (2.1) (или иначе нетривиальных продолжений операторов $a_{i}$ ) эквивалентно условию подчиненности алгебры $\mathcal{H}$ линейному функционалу $\lambda$. Таким образом, существование поляризации $\mathcal{H}$ для ковектора $\lambda$ является необходимым и достаточным условием для выполнения соотношений (2.1) и (2.2).

Покажем теперь, что свойство нормальности поляризации, или иначе выполнение $y c$ ловия Пуканского $\lambda+\mathcal{H}^{\perp} \subset O_{\lambda}$, необходимо и достаточно для выполнения соотношения (2.3).

Обозначим через $\left\{e_{A}\right\}$ базис алгебры изотропии $\mathcal{H},\left\{e_{a}\right\}$ дополнительные базисные векторы в $\mathcal{G}^{c}: e_{i}=\left\{e_{A}, e_{a}\right\}$. По определению алгебры изотропии $\alpha_{A}^{a}(0)=0$, тогда $\operatorname{det} \alpha_{a}^{b}(0) \neq 0$. Совершая линейную замену координат $q$ и базиса в $\mathcal{G}^{c}$, без потери обшности мы можем считать $\alpha_{a}^{b}(0)=\delta_{a}^{b}$. В принятых нами обозначениях $\mathcal{H}^{\perp}=\left\{\left(0, p_{a}\right)\right\}$, где $p_{a}$ - произвольные числа, условие Пуканского принимает вид $\left(\lambda_{A}, p_{a}+\lambda_{a}\right) \in O_{\lambda}$.

Пусть справедливы соотношения (2.3). Положим в (2.3) $q=0$, тогда

$$
\Phi\left(\lambda_{A}, \lambda_{a}\right)=\left.\Phi\left(f_{A}(q, p, \lambda), f_{a}(q, p, \lambda)\right)\right|_{q=0}=\Phi\left(\lambda_{A}, p_{a}+\lambda_{a}\right) .
$$

(Напоминаем обозначения: $\Phi=\left(F^{(s)}, K^{(s)}\right)$.) Из этого равенства следует, что при любом значении величин $p_{a}$ точка $\left(\lambda_{A}, p_{a}+\lambda_{a}\right)$ принадлежит тому же классу орбит, что и точка $\left(\lambda_{A}, \lambda_{a}\right)$, а т.к. класс орбит состоит из счетного числа $K$-орбит, то можно утверждать, что указанные выше две точки принадлежат одной орбите.

Обратно, пусть вьполнено условие Пуканского, что означает $f(0, p, \lambda)=\left(\lambda_{A}, p_{a}+\right.$ $\left.\lambda_{a}\right) \in O_{\lambda}$ и $\Phi\left(\lambda_{A}, p_{a}+\lambda_{a}\right)=\Phi\left(\lambda_{A}, \lambda_{a}\right)$. Покажем, что тогда имеет место формула (2.3). Поскольку функции $\Phi(f)$ удовлетворяют уравнениям (1.1), (1.3), имеем

$$
\left.\left(\alpha_{b}^{a}(q) \frac{\partial \Phi(f(q, p, \lambda))}{\partial q^{a}}-\frac{\partial f_{b}(q, p, \lambda)}{\partial q^{a}} \frac{\partial \Phi(f(q, p, \lambda))}{\partial p_{a}}\right)\right|_{q=0}=\left.\frac{\partial \Phi(f(q, p, \lambda))}{\partial q^{b}}\right|_{q=0}=0 .
$$

Таким образом, $\Phi(f(q, p, \lambda))=\Phi(f(0, p, \lambda))=\Phi(\lambda)$. Теорема 1 доказана. 
Известно, что для произвольной алгебры Ли и любого невырожденного ковектора сушествует разрешимая поляризация. С другой стороны, если алгебра $\mathcal{G}$ разрешима, то каждый функционал обладает поляризацией $\mathcal{H} \subset \mathcal{G}^{c}$.

В классическом методе орбит поляризация появляется как $n-(1 / 2) \operatorname{dim} O_{\lambda}$-мерная подалгебра $\mathcal{H} \subset \mathcal{G}^{c}$, для которой функционал $\lambda$ задает ее одномерное представление. В нашем случае нормальная поляризация определяет линейный переход (2.4) к каноническим координатам.

Нетрудно видеть, что замена функционала $\lambda$ на другой ковектор, принадлежаший той же орбите, приводит к замене поляризации $\mathcal{H}$ на сопряженную $\widetilde{\mathcal{H}}$, при этом соответствующие этим двум поляризациям координаты Дарбу связаны точечным преобразованием: $\tilde{q}^{a}=\tilde{q}^{a}(q), \tilde{p}_{a}=\left(\partial q^{b} / \partial \tilde{q}^{a}\right) p_{b}$. Таким образом, выбор конкретного представителя на орбите несуществен. Если же поляризации не сопряжены, то соответствующие координаты Дарбу связаны более общим каноническим преобразованием. Если удастся определить “квантовое" каноническое преобразование (где $q, p$ - операторы, см. ниже), то тем самым будет построен сплетающий оператор для двух представлений, полученных методом орбит с участием двух поляризаций.

Наконец, если для данного функционала не сушествует поляризации, то все равно можно построить переход к координатам Дарбу (нелинейный по переменным $p$ ) и определить понятие $\lambda$-представления алгебры $\mathcal{G}$ (см. ниже), которое является основой для гармонического анализа на группах Ли и однородных пространствах (применение метода орбит к гармоническому анализу выходит за рамки настояшей статьи и здесь не рассматривается). Иначе говоря, наличие поляризации - полезное свойство, но вовсе не обязательное для применения метода орбит.

Как уже говорилось, функционал $\lambda$ может иметь несколько различных поляризаций, однако легко проверить, что имеет место

УТВЕРЖДЕНИЕ 3. Пусть для данного $\lambda \in \mathcal{G}^{*}$ существует нормальная поляризаиия $\mathcal{H}$, тогда $\mathcal{G}_{\lambda} \subset \mathcal{H}$.

ПримеР 4 (Группа $S O(2,1)$, продолжение примера 3 ). Форма Кириллова на невырожденных орбитах имеет вид $\omega_{\lambda}=d f_{2} \wedge d f_{3} / 2 f_{1}$. Для различных спектральных типов получаем:

$$
\begin{aligned}
\text { тип } \mathrm{A}: f_{1} & =p, \quad f_{2}=e^{q}(-p+j), \quad f_{3}=e^{-q}(p+j), \\
\lambda & =(0, j, j), \quad \mathcal{H}=\left\{e_{2}+e_{3}, e_{1}+e_{2}\right\}, \quad(p, q) \in R^{2} ; \\
\text { тип В: } f_{1} & =p, \quad f_{2}=e^{q}(-i p+j), \quad f_{3}=-e^{-q}(i p+j), \\
\lambda & =(0, j,-j), \quad \mathcal{H}=\left\{e_{2}-e_{3}, e_{1}-i e_{2}\right\} .
\end{aligned}
$$

Чтобы выяснить область определения переменных $(p, q)$ для орбит типа В, разложим комплексную переменную $q$ на вещественную и мнимую компоненты: $q=\alpha+i \beta$. Используя свойство вещественности переменных $f_{i}$, получаем

$$
p=j \operatorname{tg} \beta, \quad f_{1}=j \operatorname{tg} \beta, \quad f_{2}=\frac{j e^{\alpha}}{\cos \beta}, \quad f_{3}=-\frac{e^{-\alpha}}{\cos \beta},
$$


откуда следует, что $p$ - переменная, принимаюшая любое вешественное значение, $q-$ комплексная переменная, вешественная часть которой принадлежит $R^{1}$, а мнимая $S^{1}$, т.е. $q \approx q+2 \pi i$. Таким образом, функции на $K$-орбите типа В - это аналитические функции от вешественной переменной $p$ и комплексной переменной $q$, причем по $q$ эти функции $2 \pi i$-периодичны.

Определим понятие квантования $K$-орбит. Подобное квантование проводится отдельно для каждого спектрального типа орбит и заключается в сопоставлении спектральному типу орбит специального представления алгебры Ли ( $\lambda$-представления), а на орбиты при этом накладывается условие иелочисленности, которому будет посвящен следующий раздел.

Будем рассматривать функции перехода $f_{i}(q, p, \lambda(j))$ к локальным каноническим координатам как символы операторов. Последние определяются следующим образом: переменные $p_{a}$ заменяются операторами дифференцирования $p_{a} \rightarrow \hat{p}_{a} \equiv-i \hbar \partial / \partial q^{a}$, тогда координаты ковектора $f_{i}$ переходят в линейные операторы

$$
f_{i}(q, p, \lambda(j)) \rightarrow \hat{f}_{i}\left(q,-i \hbar \frac{\partial}{\partial q}, \lambda(\tilde{j})\right)
$$

(здесь $\hbar$ - некоторый вещественный положительный параметр). Разумеется, указанная выше процедура квантования неоднозначна. Эта неоднозначность исчезает, если мы потребуем, чтобы операторы $\hat{f}_{i}$ удовлетворяли коммутационным соотношениям

$$
\frac{i}{\hbar}\left[\hat{f}_{i}, \hat{f}_{j}\right]=C_{i j}^{k} \hat{f}_{k}
$$

Если переход к каноническим координатам линейньй, т.е. для орбит этого типа сушествует нормальная поляризация, то очевидно, что

$$
\hat{f}_{i}=-i \hbar \alpha_{i}^{a}(q) \frac{\partial}{\partial q^{a}}+\chi_{i}(q, \lambda(\tilde{j}))
$$

и соотношение (2.8) полностью эквивалентно условиям $(2.6),(2.7)$.

Произвольной аналитической функции на коалгебре $\varphi(f)$ при квантовании сопоставляется симметризованная операторная функция $\varphi(\hat{f})$ от операторов $\hat{f}_{i}$. Параметры $\tilde{j}$ связаны с параметрами орбиты $j$ следуюшим образом: $\tilde{j}=j+i \hbar \beta$, где $\beta$ является $r_{(s)}$-мерным вещественным вектором, определяемым из условия вешественности функций

$$
\kappa_{\mu}^{(s)}(j)=K_{\mu}^{(s)}(\hat{f})
$$

Отметим, что в “классическом" пределе $\hbar \rightarrow 0$ мы имеем $\kappa_{\mu}^{(s)}(j) \rightarrow \omega_{\mu}^{(s)}(j)$ и коммутатор линейных операторов переходит в скобку Пуассона на коалгебре

$$
\frac{i}{\hbar}[\cdot, \cdot] \rightarrow\{\cdot, \cdot\}
$$

3 Теоретическая и математическая физика, т. 123, № 3, 2000 г. 
Так как функции $\kappa_{\mu}^{(s)}(j)$ при $\hbar \neq 0$ в общем случае отличаются от функций $\omega_{\mu}^{(s)}(j)$, то мы должны переопределить области определения $J^{(s)}$ параметров $j$ так, чтобы каждой точке $j \in J^{(s)}$ взаимно однозначно соответствовали бы значения функций $\kappa^{(s)}(j)$, т.е. при ограничении на $J^{(s)}$ функции $\left(\kappa^{(s)}\right)^{-1}$ были бы однозначны. При этом должно вьполняться условие

$$
\kappa^{(s)}\left(J^{(s)}\right)=\Omega^{(s)} .
$$

Введем операторы

$$
l_{k}\left(q, \partial_{q}, j\right) \equiv \frac{i}{\hbar} \hat{f}_{k}(q, \hat{p}, \lambda(\tilde{j}))
$$

Очевидно, что

$$
\begin{gathered}
{\left[l_{i}, l_{j}\right]=C_{i j}^{k} l_{k}, \quad F_{\alpha}^{(s)}\left(-i \hbar l\left(q, \partial_{q}, j\right)\right) \equiv 0, \quad K_{\mu}^{(s)}\left(-i \hbar l\left(q, \partial_{q}, j\right)\right) \equiv \kappa_{\mu}^{(s)}(j),} \\
\kappa_{\mu}^{(s)}(j)=\overline{\kappa_{\mu}^{(s)}(j)}, \quad \operatorname{det} \frac{\partial \kappa_{\mu}^{(s)}(j)}{\partial j_{\nu}} \neq 0, \quad j \in J, \quad q \in Q .
\end{gathered}
$$

ОПРЕДЕЛЕНИЕ 1 . Пусть $f_{i}=f_{i}(q, p, \lambda(j))$ - переход к каноническим координатам на орбите $O_{\lambda(j)}, \lambda(j)$ - параметризованный ковектор. Реализацию алгебры Ли $\mathcal{G}$ соответствуюшими операторами $l_{i}\left(q, \partial_{q}, j\right)$ будем называть $\lambda$-представлением.

Ниже мы покажем, что величины $\kappa_{\mu}^{0}(j)$ образуют спектр операторов Казимира на группе Ли $\widehat{K}_{\mu} \equiv K_{\mu}(i \hbar \xi(g))$ (здесь $\xi_{i}(g)$ - левоинвариантные векторные поля на группе $G)$. Соответственно $\kappa_{\mu}^{(s)}(j)$ - собственные значения операторов Казимира $\widehat{K}_{\mu}^{(s)} \equiv$ $K_{\mu}^{(s)}(i \hbar X)$ (здесь $X_{i}$ - генераторы группы преобразований) на однородном пространстве $(s)$-типа $[5]$.

ПримеР 5 (Группа $S O(2,1)$, продолжение примера 3$)^{1)}$.

$$
\begin{gathered}
\text { Тип } \mathrm{A}: \hat{f}_{1}=-i \hbar \frac{\partial}{\partial q}, \quad \hat{f}_{2}=e^{q}\left(i \hbar \frac{\partial}{\partial q}+j+i \hbar \beta\right), \quad \hat{f}_{3}=e^{-q}\left(-i \hbar \frac{\partial}{\partial q}+j+i \hbar \beta\right), \\
\kappa(j)=K(\hat{f})=\hat{f}_{1}^{2}+\hat{f}_{2} \circ \hat{f}_{3}=j^{2}+\hbar^{2} \beta(1-\beta)+i \hbar j(2 \beta-1) .
\end{gathered}
$$

Из условия вешественности $\kappa(j)$ получаем $\beta=1 / 2$. Тогда $\lambda$-представление спектрального типа А выглядит следующим образом:

$$
l_{1}=\frac{\partial}{\partial q}, \quad l_{2}=e^{q}\left(-\frac{\partial}{\partial q}+\frac{i}{\hbar} j-\frac{1}{2}\right), \quad l_{3}=e^{-q}\left(\frac{\partial}{\partial q}+\frac{i}{\hbar} j-\frac{1}{2}\right), \quad \kappa(j)=j^{2}+\frac{\hbar^{2}}{4},
$$

причем $j \in J^{\mathrm{A}}=[0, \infty), \Omega^{\mathrm{A}} \equiv \kappa\left(J^{A}\right)=\left[\hbar^{2} / 4, \infty\right)$.

Тип В: $\hat{f}_{1}=-i \hbar \frac{\partial}{\partial q}, \quad \hat{f}_{2}=e^{q}\left(-\hbar \frac{\partial}{\partial q}+j+i \hbar \beta\right), \quad \hat{f}_{3}=e^{-q}\left(-\hbar \frac{\partial}{\partial q}-j-i \hbar \beta\right)$,

$$
\kappa(j)=K(\hat{f})=\hat{f}_{1}^{2}+\hat{f}_{2} \circ \hat{f}_{3}=-j^{2}+\hbar^{2} \beta^{2}-\hbar j-i \hbar \beta(2 j+\hbar) .
$$

1) Здесь и ниже знак "о" означает симметризованное произведение операторов $A \circ B \equiv(A B+$ $B A) / 2$. 
Из условия вешественности $\kappa(j)$ получаем $\beta=0 ; \lambda$-представление спектрального типа В имеет вид

$$
l_{1}=\frac{\partial}{\partial q}, \quad l_{2}=e^{q}\left(-i \frac{\partial}{\partial q}+\frac{i}{\hbar} j\right), \quad l_{3}=e^{-q}\left(-i \frac{\partial}{\partial q}-\frac{i}{\hbar} j\right), \quad \kappa(j)=-j(j+\hbar) .
$$

Здесь $j \in J^{\mathrm{B}}=[-\hbar / 2, \infty), \Omega^{\mathrm{B}} \equiv \kappa\left(J^{\mathrm{B}}\right)=\left(-\infty, \hbar^{2} / 4\right)$. Условие (2.10) выполняется: $\Omega^{\mathrm{A}} \cup \Omega^{\mathrm{B}}=\Omega=R^{1}$.

Пример 6 (Группа $S t(1, R))$. Эта группа приведена Кирилловым в [3, с. 261] в качестве примера, иллюстрируюшего отсутствие поляризации для произвольного ковектора. Опишем структуру $K$-орбит этой группы.

Алгебра Ли этой группы может быть реализована матрицами

$$
X(a, \xi, c)=\left(\begin{array}{cccc}
0 & \xi_{1} & \xi_{2} & c \\
0 & a_{1} & a_{2} & \xi_{2} \\
0 & a_{3} & -a_{1} & -\xi_{1} \\
0 & 0 & 0 & 0
\end{array}\right) \equiv a_{1} e_{1}+a_{2} e_{2}+a_{3} e_{3}+\xi_{1} e_{4}+\xi_{2} e_{5}+c e_{6} .
$$

Базисные элементы $e_{i}$ имеют следуюшие ненулевые коммутационные соотношения:

$$
\begin{aligned}
& {\left[e_{1}, e_{2}\right]=2 e_{2}, \quad\left[e_{1}, e_{3}\right]=-2 e_{3}, \quad\left[e_{1}, e_{4}\right]=-e_{4}, \quad\left[e_{1}, e_{5}\right]=e_{5},} \\
& {\left[e_{2}, e_{3}\right]=e_{1}, \quad\left[e_{2}, e_{4}\right]=-e_{5}, \quad\left[e_{3}, e_{5}\right]=-e_{4}, \quad\left[e_{4}, e_{5}\right]=2 e_{6} .}
\end{aligned}
$$

Приведем описание орбит (1.4) для данной группы:

$$
\begin{aligned}
O_{\omega}^{0} & =\left\{K_{1}(f)=\omega_{1}^{0}, \quad K_{2}(f) \equiv f_{6}=\omega_{2}^{0}, \quad \neg\left(F^{1}(f)=0\right)\right\}, \\
O_{\omega}^{1 a} & =\left\{F_{1}^{1 a}(f)=F_{2}^{1 a}(f)=F_{3}^{1 a}(f)=0, \quad f_{6}=\omega^{1 a} \neq 0\right\}, \\
O_{\omega}^{1 b} & =\left\{f \neq 0, \quad f_{4}=f_{5}=f_{6}=0, \quad \frac{f_{2} f_{3}+f_{1}^{2}}{4}=\omega^{1 b}\right\}, \\
O^{2} & =\left\{f=0, \quad K_{1}(f) \equiv f_{1}^{2} f_{6}-f_{1} f_{4} f_{5}+f_{4}^{2} f_{2}-f_{5}^{2} f_{3}+4 f_{2} f_{3} f_{6}\right\}, \\
F_{1}^{1 a}(f) & \equiv 2 f_{1} f_{6}-f_{4} f_{5}, \quad F_{2}^{1 a}(f) \equiv 4 f_{2} f_{6}-f_{5}^{2}, \quad F_{3}^{1 a}(f) \equiv 4 f_{3} f_{6}+f_{4}^{2} .
\end{aligned}
$$

Нетрудно видеть, что для вырожденных орбит $O_{\omega}^{1 a}$ поляризации не существует. Форма Кириллова на орбитах этого типа выглядит следующим образом: $\omega_{\lambda}=d f_{4} \wedge d f_{5} / 2 f_{6}$. Пространство $M_{1 a}$ является пространством одного спектрального типа, и можно ввести глобальные координаты на $B_{1 a}$, т.е. параметризацию $\lambda(j)=(0,0,0,0,0, j)$. Для этого функционала имеем $\mathcal{G}_{\lambda}=\left\{e_{1}, e_{2}, e_{3}, e_{6}\right\}, \mathcal{N}_{\lambda}=\left\{e_{1}, e_{2}, e_{3}\right\}, \mathcal{K}_{\lambda}=\left\{e_{6}\right\}$. Для рассматриваемых орбит вместо линейного перехода (2.4) существует квадратичный по переменным $p$ переход к каноническим координатам

$$
f_{1}=q p, \quad f_{2}=q^{2} j, \quad f_{3}=-\frac{p^{2}}{4 j}, \quad f_{4}=p, \quad f_{5}=2 q j, \quad f_{6}=j, \quad(p, q) \in R^{2} .
$$

Построим соответствующее $\lambda$-представление. Проблемы с упорядочиванием операторов $\hat{p}=-i \hbar \partial_{q}$ и $\hat{q}=q$ в операторах $\hat{f}_{2}=q^{2} j, \hat{f}_{3}=-\hat{p}^{2} / 4 j, \hat{f}_{4}=\hat{p}, \hat{f}_{5}=2 q j, \hat{f}_{6}=j$ не возникает. Оператор $\hat{f}_{1}$ однозначно находится из коммутационных соотношений $\hat{f}_{1}=$ $q \hat{p}-i \hbar / 2$. Итак, $\lambda$-представление для орбит этого типа имеет вид

$l_{1}=q \partial_{q}+\frac{1}{2}, \quad l_{2}=\frac{i}{\hbar} q^{2} j, \quad l_{3}=\frac{i \hbar}{4 j} \partial_{q}^{2}, \quad l_{4}=\partial_{q}, \quad l_{5}=\frac{i}{\hbar} 2 q j, \quad l_{6}=\frac{i}{\hbar} j, \quad \kappa^{1 a}(j)=j \in R^{1}$. 


\section{3. УСЛОВИЕ ЦЕЛОЧИСЛЕННОСТИ ОРБИТ. СПЕКТРЫ ОПЕРАТОРОВ КАЗИМИРА}

В этом разделе описан первый этап явного построения гармонического анализа на однородных пространствах. Однако эта сложная тема заслуживает отдельного исследования, и здесь мы ее практически не затрагиваем. В данном разделе мы покажем, что введенные выше функции $\kappa(j)$ являются собственными значениями операторов Казимира, где на параметры $j$ наложены условия целочисленности.

Введем на связной и односвязной вещественной группе Ли $G$ квазиинвариантную меpy $d g=\sqrt{d_{l} g d_{r} g}$, где $d_{l} g, d_{r} g$ - левая и правая меры Хаара соответственно. Определим в пространстве $L_{2}(G, d g)$ унитарное представление группы $G \times G$ :

$$
T_{\left(g_{1}, g_{2}\right)} u(g)=\sqrt{\frac{d\left(g_{1}^{-1} g g_{2}\right)}{d g}} u\left(g_{1}^{-1} g g_{2}\right), \quad u(g) \in L_{2}(G, d g) .
$$

Инфинитезимальными генераторами представления (3.1) являются лево- $\left(\xi_{i}\right)$ и правоинвариантные $\left(\eta_{i}\right)$ операторы:

$$
\xi_{i}(g)=\xi_{i}^{j}(g) \partial_{g^{j}}+C_{i}, \quad \eta_{i}(g)=\eta_{i}^{j}(g) \partial_{g^{j}}+C_{i}, \quad C_{i} \equiv-\frac{1}{4} C_{i j}^{j}
$$

Генераторы $\xi_{i}, \eta_{i}$ отличаются на аддитивные постоянные от соответствующих лево- и правоинвариантных векторных полей на группе $G$ (а в случае унимодулярной группы совпадают с последними), и поэтому мы их также будем называть векторными полями.

Поскольку собственные функции неограниченных операторов с непрерывными спектрами являются не элементами из $L_{2}(G, d g)$, а лишь линейными функционалами на плотном множестве (ядерном пространстве) $\Phi \subset L_{2}(G, d g)$, то следует ввести триплет Гельфанда $\Phi \subset L_{2}(G, d g) \subset \Phi^{\prime}$, где $\Phi^{\prime}$ - пространство, дуальное к $\Phi$.

Аналогично разложению (1.4) разложим пространство $L_{2}(G, d g)$ на инвариантные относительно представления (3.1) подпространства $L_{2}(G, d g)=\bigcup_{(s)} \mathcal{L}_{(s)}$, где

$$
\mathcal{L}_{(s)}=\left\{\varphi(g) \in L_{2}(G, d g) \mid F_{\alpha}^{(s)}(\xi) \varphi(g)=0, \quad \neg\left(F^{s+1}(\xi) \varphi(g)=0\right)\right\} .
$$

Отметим, что замена в выражении (3.2) левоинвариантных полей $\xi_{i}$ на правоинвариантные $\eta_{i}$ не меняет пространств $\mathcal{L}_{(s)}$. (Это можно увидеть, например, совершая отображение $g \rightarrow g^{-1}$, при котором левоинвариантные поля переходят в правоинвариантные и наоборот, а мера $d g$ инвариантна.) Для каждого $\mathcal{L}_{(s)}$ также введем триплет Гельфанда $\Phi_{(s)} \subset \mathcal{L}_{(s)} \subset \Phi_{(s)}^{\prime}$

На каждом пространстве $\mathcal{L}_{(s)}$ сушествуют двухсторонне инвариантные операторы Казимира $K_{\mu}^{(s)}(i \hbar \xi)=K_{\mu}^{(s)}(-i \hbar \eta)$ и пространство $\mathcal{L}_{(s)}$ разлагается в прямую сумму (интеграл) собственных подпространств операторов Казимира, т.е. имеется полная аналогия с разложением (1.4). 
TЕОРема 2. Пусть $\lambda(j)$ - параметризованный $(s)$-ковектор, тогда величины $\kappa_{\mu}^{(s)}(j)(2.9)$ являются собственными значениями для операторов Казимира $K_{\mu}^{(s)}(i \hbar \xi)$ на $\mathcal{L}_{(s)}$, әде параметры $j$ удовлетворяют условию

$$
\left\langle\lambda(j), e_{\mu}\right\rangle=\frac{2 \pi \hbar n_{\mu}}{T_{\mu}}, \quad n_{\mu} \in Z .
$$

Здесь $e_{\mu}-$ базисный вектор одномерной алгебры Ли однопараметрической компактной подгруппы коммутативной фактор-группы $K_{\lambda}, T_{\mu}$ - период одномерной компактной подгруппь $\left(\exp \left(T_{\mu} e_{\mu}\right)=1\right)$.

Таким образом, для определения спектров операторов Казимира достаточно найти функции $\kappa_{\mu}^{(s)}(j)$ (эти функции фактически задаются структурными константами и определяют спектр операторов Казимира на универсальной накрываюшей группе $\widetilde{G}$ ) и наложить на параметры $j$ условия квантования (3.3). Чуть ниже мы покажем, что условие (3.3) эквивалентно условию челочисленности $K$-орбит Кириллова.

ДОКАЗАТЕЛЬСТво ТЕОРЕМЫ 2. Обсудим основные моменты доказательства. Пусть $\lambda(j)$ - параметризованный $(s)$-ковектор, $l_{i}\left(q, \partial_{q}, j\right)$ - соответствующее $\lambda$-представление. Определим обобщенные функции $D_{q}^{j}(g)$ на группе $G$ уравнениями

$$
\left[\xi_{i}(g)+l_{i}\left(q, \partial_{q}, j\right)\right] D_{q}^{j}(g)=0, \quad i=1, \ldots, n .
$$

Из определения $\lambda$-представления (2.11) легко получить

$$
F_{\alpha}^{(s)}(\xi) D_{q}^{j}(g)=0, \quad \text { т.е. } D_{q}^{j}(g) \in \Phi_{(s)}^{\prime}, \quad K_{\mu}^{(s)}(i \hbar \xi) D_{q}^{j}(g)=\kappa_{\mu}^{(s)}(j) D_{q}^{j}(g) .
$$

Несмотря на то что система (3.4) совместна, функции $D_{q}^{j}(g)$ глобально на всей группе сушествуют не при любых значениях параметров $j$. Ограничим систему (3.4) на подгруппу $G_{\lambda}$ и положим $q=0$. Тогда с учетом утверждения 1 получим

$$
\left[\xi_{A}\left(g_{\lambda}\right)+\frac{i}{\hbar} \lambda_{A}(\tilde{j})\right] D_{0}^{j}\left(g_{\lambda}\right)=0, \quad g_{\lambda} \in G_{\lambda},
$$

где индекс $A$ нумерует базисные векторы алгебры $\mathcal{G}_{\lambda}$. Из $(3.5)$ следует, что функции $D_{q}^{j}(g)$ определены глобально лиш при выполнении условия

$$
\frac{1}{2 \pi \hbar} \oint_{\gamma \in H_{1}\left(G_{\lambda}\right)} \omega_{G_{\lambda}}^{j}=n_{\gamma} \in Z
$$

Здесь $\omega_{G_{\lambda}}^{j}=\omega^{A} \lambda_{A}(j)$ - замкнутая левоинвариантная 1-форма на группе $G_{\lambda}$. (Фактически мы переоткрыли критерий Костанта [8].) Условие (3.6) эквивалентно условию челочисленности орбит Кириллова [3].

С помощью полученных в предыдущих разделах результатов можно представить условие целочисленности (3.6) в более развернутом виде. 
Для выбранного параметризованного ковектора $\lambda(j)$ базис левоинвариантных векторных полей алгебры $\mathcal{G}_{\lambda}$ образован векторами $(\tilde{\lambda} \equiv \lambda(\tilde{j}))$

$$
\begin{aligned}
\nabla F_{\alpha}^{(s)}(g) & \equiv\left(L_{g}\right)_{*} \nabla F_{\alpha}^{(s)}(\tilde{\lambda})=F_{\alpha}^{(s) i}(\tilde{\lambda}) \xi_{i}(g), \\
\nabla K_{\mu}^{(s)}(g) & \equiv\left(L_{g}\right)_{*} \nabla K_{\mu}^{(s)}(\tilde{\lambda})=K_{\mu}^{(s) i}(\tilde{\lambda}) \xi_{i}(g),
\end{aligned}
$$

где

$$
F_{\alpha}^{(s) i}(\tilde{\lambda})=\left.\frac{\partial F_{\alpha}^{(s)}(f)}{\partial f_{i}}\right|_{f=\tilde{\lambda}}, \quad K_{\mu}^{(s) i}(\tilde{\lambda})=\left.\frac{\partial K_{\mu}^{(s)}(f)}{\partial f_{i}}\right|_{f=\tilde{\lambda}},
$$

$L_{g}$ - левоинвариантное представление группы $G \times G$ на пространстве $L_{2}(G, d g)$. Перепишем систему (3.5) следуюшим образом:

$$
\begin{gathered}
\nabla F_{\alpha}^{(s)}\left(g_{\lambda}\right) D_{0}^{j}\left(g_{\lambda}\right)=0, \quad g_{\lambda} \in G_{\lambda} \\
{\left[\nabla K_{\mu}^{(s)}\left(g_{\lambda}\right)+\frac{i}{\hbar} m_{\mu}^{(s)} \omega_{\mu}^{(s)}(\tilde{j})\right] D_{0}^{j}\left(g_{\lambda}\right)=0, \quad g_{\lambda} \in G_{\lambda} .}
\end{gathered}
$$

Операторы $\nabla F_{\alpha}^{(s)}$ в формуле (3.7) представляют собой левоинвариантные векторные поля на нормальной подгруппе $N_{\lambda}$, а значит, функция $D_{0}^{j}\left(g_{\lambda}\right)$ не зависит от координат элемента $n$ в разложении $g_{\lambda}=n k, n \in N_{\lambda}, k \in K_{\lambda}$. Аналогично операторы $\nabla K_{\mu}^{(s)}$ являются левоинвариантными векторными полями на коммутативной группе $K_{\lambda}$, а система (3.8) тем самым представляет собой задачу на собственные значения для $r_{(s)}$ коммутирующих операторов первого порядка. Последняя решается тривиально. Очевидно, что если однопараметрическая подгруппа с генератором $\nabla K_{\mu}^{(s)}$ некомпактна, то соответствуюшее собственное число $m_{\mu}^{(s)} \omega_{\mu}^{(s)}(\tilde{j})$ может принимать любое значение, т.е. параметры $j$ в этом случае не квантуются. Если выбрать базис, не зависящий от параметров $j$ (а такой базис вследствие однородности функций Казимира всегда существует), то система (3.8) для компактных подгрупп в $K_{\lambda}$ примет вид

$$
\left[\frac{\partial}{\partial k^{\mu}}+\frac{i}{\hbar} \lambda_{\mu}(j)\right] D_{0}^{j}(k)=0
$$

Таким образом, условие целочисленности дается формулой (3.3).

Итак, мы показали, что функции $\kappa_{\mu}^{(s)}(j)$ от целочисленных параметров $j$ принадлежат спектру операторов Казимира на $\mathcal{L}_{(s)}$. Для завершения доказательства необходимо показать, что семейство функций $D_{q}^{j}(g)$ плотно в $\Phi_{(s)}^{\prime}$, что, в свою очередь, означает отсутствие других элементов спектра. Есть все основания считать, что дело обстоит именно так [5], однако обсуждение этого вопроса выводит нас далеко за рамки темы настоящей работы. 
ПримеР 7 (Группа $S O(2,1)$, продолжение примера 3$)$. Для спектрального типа А: $\lambda(j)=(0, j, j), \mathcal{G}_{\lambda}=\left\{e_{2}+e_{3}\right\} ;$ для спектрального типа В: $\lambda(j)=(0, j,-j), \mathcal{G}_{\lambda}=\left\{e_{2}-\right.$ $\left.e_{3}\right\}$. Таким образом, для выделения целочисленных орбит нам необходимо знать, являются ли соответствующие однопараметрические группы компактными или нет. Очевидно, что для спектрального типа А группа $K_{\lambda}=\exp \left[(1 / 2) t\left(e_{2}+e_{3}\right)\right]$ некомпактна и, таким образом, все орбиты этого типа целочисленны, т.е. параметр $j$ не квантуется; $\kappa(j)=j^{2}+\hbar^{2} / 4, j \geqslant 0$. Для спектрального типа В группа $K_{\lambda}=\exp \left[(1 / 2) t\left(e_{2}-e_{3}\right)\right]$ компактна и имеет период $T=2 \pi$. Из формулы (3.3) имеем $\left\langle\lambda(j),(1 / 2)\left(e_{2}-e_{3}\right)\right\rangle=j=n \hbar$, откуда $j=n \hbar, n \in Z$ и $\kappa(j)=-\hbar^{2} n(n+1), n=0,1, \ldots$.

\section{Список литературы}

[1] А.А. Кириллов. УМН. 1962. Т. 17. № 4. С. 57-110.

[2] А. А. Кириллов. Функц. анализ и его прилож. 1968. Т. 2. № 2. С. 40-55; 1969. Т. 3. № 1. C. $36-47$.

[3] А.А. Кириллов. Элементы теории представлений. М.: Наука, 1978.

[4] A.A. Кириллов. Введение в теорию представлений и некоммутативный гармонический анализ. В сб.: Итоги науки и техн. Соврем. проблемы математики. Фундам. напр. Т. 22. Теория представлений и некоммутативный гармонический анализ. Ред. Р.В.Гамкрелидзе. М.: ВИНИТИ, 1988. С. 5-162.

[5] И. В. Широков. K-орбиты, гармонический анализ на однородных пространствах и интегрирование дифференциальных уравнений. Препринт. Омск: ОмГУ, 1998.

[6] А. В. Шаповалов, И. В. Широков. ТМФ. 1995. Т. 104. № 2. С. 195-213.

[7] А. С. Мищенко, А. Т. Фоменко. Тр. семин. вектор. тензор. анал. 1979. Т. 19. С. 3-94.

[8] B. Kostant. Quantization and Unitary Representations. I. Prequantization. In: Lectures in Modern Analysis and Applications, III. Ed. C.T. Taam. Berlin: Springer-Verlag, 1970. P. 87-208. 